\title{
ORIGINALS
}

\section{A Study of the Morphological Changes in the Small Intestine of the Spontaneously Diabetic Chinese Hamster*}

\author{
A. R. Diani**, G. C. Gerritsen, S. Stromsta and P. Murray \\ Biology Dept. Waco, Texas, Kalamazoo and Diabetes and Atherosclerosis Research, The Upjohn Co., Kalamazoo, MI, USA
}

\begin{abstract}
Summary. Several morphological changes were observed microscopically in the small intestine of some diabetic Chinese hamsters. Although some alterations lacked statistical significance due to variation, most diabetics displayed a greater incidence and severity compared with nondiabetic controls. The following structural deviations were seen in the small intestines of some diabetics: increased surface area, elevated number of goblet cells per villus, decreased muscle thickness with connective tissue infiltration, reduced number of Auerbach's plexuses, lymphocyte aggregations accompanied by blunted villi, blood vascular lesions and deformed villi due to excessive loss of epithelial cells.
\end{abstract}

Key words: Chinese hamster, small intestine, diabetic, villi, goblet cells, Auerbach's plexus, lymphocyte aggregations, blood vascular lesions, epithelial cell loss.

During the last few years, a number of organ systems of the hamster (Cricetulus griseus) have been studied morphologically. These investigations have focused on the pancreas, retina, kidney [1], peripheral nerves [2], brain [3], testes, skin [4] and aorta [5]. Surprisingly, no structural studies were done on the gastrointestinal tube of the diabetic Chinese hamster.

Therefore, a light microscopic examination of the small intestine of the diabetic Chinese hamster was undertaken. It was felt that this organ was appropriate to study because of the following factors: 1 . elevated

\footnotetext{
* Supported in part by Upjohn Company grant

** Supported in part by Western Michigan University Summer Faculty Fellowship (1974)
}

free fatty acid absorption [6], 2. steatorrhea and hyperphagia [7], 3. hormone inbalances [8,9], 4. similar origin and development of the pancreas and small intestine, and 5 . previously described disorders associated with the small intestine of human diabetics $[10,11,12,13,14,15,16,17]$. Morphological alterations in the small bowel of the diabetic Chinese hamster will be described as a basis for more extensive gastrointestinal studies in the future.

\section{Material and Methods}

The animals for this investigation were selected from the Upjohn colony of Chinese hamsters [9]. A total of 15 pairs of animals were used. Each pair consisted of a nonketotic diabetic matched with a nondiabetic. The nonketotic diabetics were taken from 7 different inbred sublines, whereas the nondiabetics were derived from 3 sublines. Each pair was matched for body weight, sex and age. Mean body weight of the diabetics was $33 \pm 2$ grams (range 27-37) and the mean for nondiabetics was $32 \pm 2$ grams (range 26-38). Age varied from 8 to 18 months and duration of diabetes from 8 to 16 months. All diabetic hamsters displayed continuous glycosuria as measured by consistent TesTape ${ }^{\circledR}$ values of plus 3 to plus 4 (with one exception) and negative Ketostix ${ }^{\circledR}$ values for urine ketones. TesTape values were previously reported to be acceptable measures of diabetes in the Chinese hamster [18]. Blood samples were drawn from the orbital sinus just prior to sacrifice and analyzed for glucose by the Auto-Analyzer technique. All animals were fed Purina Mouse Breeder Chow and allowed food and water ad libitum until sacrifice. None of the diabetic hamsters received insulin therapy at any time.

Immediately after sacrifice, the small intestine was removed in its entirety from just distal to the pyloric 
Table 1. Microscopic measurements

\begin{tabular}{|c|c|c|c|c|c|c|c|c|}
\hline \multirow[b]{3}{*}{ Structure } & \multicolumn{4}{|c|}{ Proximal Third } & \multicolumn{4}{|c|}{ Middle Third } \\
\hline & \multicolumn{2}{|c|}{ Anterior } & \multicolumn{2}{|c|}{ Posterior } & \multicolumn{2}{|c|}{ Anterior } & \multicolumn{2}{|c|}{ Posterior } \\
\hline & $\overline{\text { Diab. }}$ & Nondiab. & Diab. & Nondiab. & Diab. & Nondiab. & Diab. & Nondiab. \\
\hline Villi/Cross-Sect. & $35^{\mathrm{a}}$ & 29 & 31 & 28 & $42^{a}$ & 38 & $48^{\mathrm{b}}$ & 42 \\
\hline Cross-Sect. Area $\left(\mathrm{mm}^{2}\right)$ & $3.6^{\mathrm{b}}$ & 3.0 & 3.9 & 3.5 & $4.0^{\mathrm{a}}$ & 3.4 & $4.7^{\mathrm{b}}$ & 3.4 \\
\hline Villi Length $(\mu)$ & $413^{\mathrm{a}}$ & 373 & 462 & 447 & 447 & 438 & 357 & 348 \\
\hline Villi width (Base) $(\mu)$ & 89 & 90 & 100 & 93 & 98 & 95 & $96^{\mathrm{c}}$ & 84 \\
\hline Villi Width (Middle) $(\mu)$ & 82 & 82 & 87 & 81 & $89^{c}$ & 80 & $94^{\mathrm{b}}$ & 81 \\
\hline Villi width (Tip) $(\mu)$ & 66 & 64 & 60 & 54 & $61^{\mathrm{c}}$ & 55 & $71^{\mathrm{c}}$ & 61 \\
\hline Goblet Cells/Villus & $11^{\mathrm{b}}$ & 9 & $12^{\mathrm{b}}$ & 9 & 11 & 10 & 10 & 10 \\
\hline
\end{tabular}

${ }^{a} \mathbf{P}<0.1$ Diab vs. Nondiab

${ }^{b} \mathrm{P}<0.05$ Diab vs. Nondiab

${ }^{c} \mathbf{P}<0.01$ Diab vs. Nondiab

Table 2. Incidence of pathological observations in diabetics

\begin{tabular}{lllll}
\hline Structure & \multicolumn{2}{l}{$\%$ Incidence } \\
\cline { 2 - 5 } & \multicolumn{2}{c}{ Proximal Third } & \multicolumn{2}{c}{ Middle Third } \\
\cline { 2 - 5 } & \multicolumn{3}{c}{ Anterior Posterior } & \multicolumn{2}{c}{ Anterior Posterior } \\
Reduced Muscle & 47 & 47 & 14 & 20 \\
Reduced Auerbach's & & & & \\
Plexuses & 33 & 47 & 20 & 14 \\
Lymphocyte Aggrega- & 40 & 33 & 6 & 6 \\
tions & & & & \\
Blood Vascular Lesions & 0 & 27 & 0 & 0 \\
Vasodilation & 0 & 0 & 53 & 46 \\
Epithelial Loss & 27 & 13 & 25 & 53 \\
\hline
\end{tabular}

stomach to the ileocaecal valve. Due to the fact that there were no previous histological or anatomical guidelines concerning the structure or relative location of the duodenum, jejunum or ileum of the Chinese hamster, the intestine was arbitrarily divided into thirds. This report concerns the initial two thirds of the small intestine. Serial cross sections were prepared and $90 \%$ of these sections were examined. For comparative purposes, similar tissue sections from diabetic and control intestines were studied. Tissues were fixed and stained according to standardized histological procedures [19].

Quantitative study of the diabetic and control intestines was accomplished by measuring several structures with a micrometer. The measurements of structures are presented in Table 1. Pathological observations were recorded as percent incidence in diabetics as shown in Table 2.

Since frequency of measurements and structural changes varied in different areas, the data were organized into 4 regions of the intestine, the anterior and posterior regions of both the proximal and middle third.

\section{Results}

Non-fasting blood sugars of 8 out of the 15 pairs were obtained. Mean blood sugar of the diabetics was 244 \pm 18 milligrams percent (range 145-320) and the mean for nondiabetics was $107 \pm 6$ milligrams percent (range 73-125).

On the basis of microscopic structure, there were no discrete morphological characteristics common to any region of the small intestine which would classify it in a classical manner. The standard morphological characteristics of the mammalian small intestine were observed in the Chinese hamster with the exception of plicae circulares, Brunner's glands, and a muscularis mucosae.

There were two conditions which were observed grossly in the small intestines of most of the diabetic hamsters that were not apparent in any of the small bowels of the nondiabetics. The small intestines of the diabetic hamsters displayed some distension which was attributed to excessive accumulation of partially digested food material. The small bowels of the diabetic hamsters were soft and friable in contrast to firmness of the control intestines.

\section{Microscopic Measurements}

Significant microscopic measurements are presented in Table 1. The number of villi per cross-section and cross-sectional area was significantly increased in the diabetics except for the posterior portion of the proximal third. Extensive damage to villi was observed. Therefore, measurements were made only on normal appearing villi of the diabetics. However, size of normal appearing villi of the diabetics appeared to be larger. The number of goblet cells per villus was significantly higher in both portions of the proximal third of the diabetic intestines. 


\section{Histopathology}

Incidence of pathological observations is recorded in Table 2. Reduction of the muscular coat (Fig. 4) was observed more frequently in the proximal third of the intestines of some diabetics. For comparative purposes, the nondiabetic is shown in Fig. 3. It should be pointed out that considerable variation in thickness of the muscle was observed in diabetics. In some instances, fibrous connective tissue infiltration was widespread throughout the external layer of the slender muscularis of the diabetic intestines. Other muscular pathologies included pyknosis of some of the muscle nuclei and degeneration of the muscularis. The last two findings were highly variable and were seen primarily in a few of the diabetic intestines.

Reduction of Auerbach's plexuses was observed more frequently in the proximal third of the diabetic intestines (Table 2). Extensive areas of diabetic intestines were devoid of Auerbach's plexuses (Fig. 4) but they were frequently observed in nondiabetic controls (Fig. 3). Although not shown, the neuron cell bodies associated with the plexuses of Auerbach exhibited some irregularities in a few of the diabetic intestines. These included shrinkage of the cell bodies, pyknosis of the nuclei and non-uniform staining of the neuroplasm. Lymphocyte aggreations were increased in the proximal third of the diabetic intestines (Table 2). Massive, dense collections of lymphocytes (Fig. 2) thoroughly infiltrated the submucosa and mucosa of some diabetic intestines. For comparative purposes, the nondiabetic is shown in Figure 1. Some aggregates were highly developed and penetrated approximately one-half of the circumference of the intestines, but others were not as extensive. Furthermore, wandering cells or macrophages (Fig. 6) were present among the lymphocytes. Pronounced morphological changes were detectable in the areas of the intestines where lymphocyte aggregates were observed. Some of the long, thin fingerlike villi were transformed into blunted or flattened processes (Fig. 2). The simple columnar epithelia of some of the villi were modified into a pseudostratified or stratified squamous condition. There was marked thickening of the connective tissue of the tunica propria and submucosa (Fig. 2). It should be emphasized that aggregates of lymphocytes were detected in the nondiabetic intestines. However, they never approached the dimensions of those in the diabetic intestines.

It is of considerable interest that blood vascular lesions were confined solely to the posterior portions of the proximal third of the diabetic intestines (Table 2). These vessels were extremely distended (Fig. 6) and were situated in the submucosal tissue. The vascular walls were usually thickened and appeared to be considerably irregular or damaged. Deposits of hyaline material were localized in the lumina of all the pathological vessels. Controlvessels are shown inFig. 5.

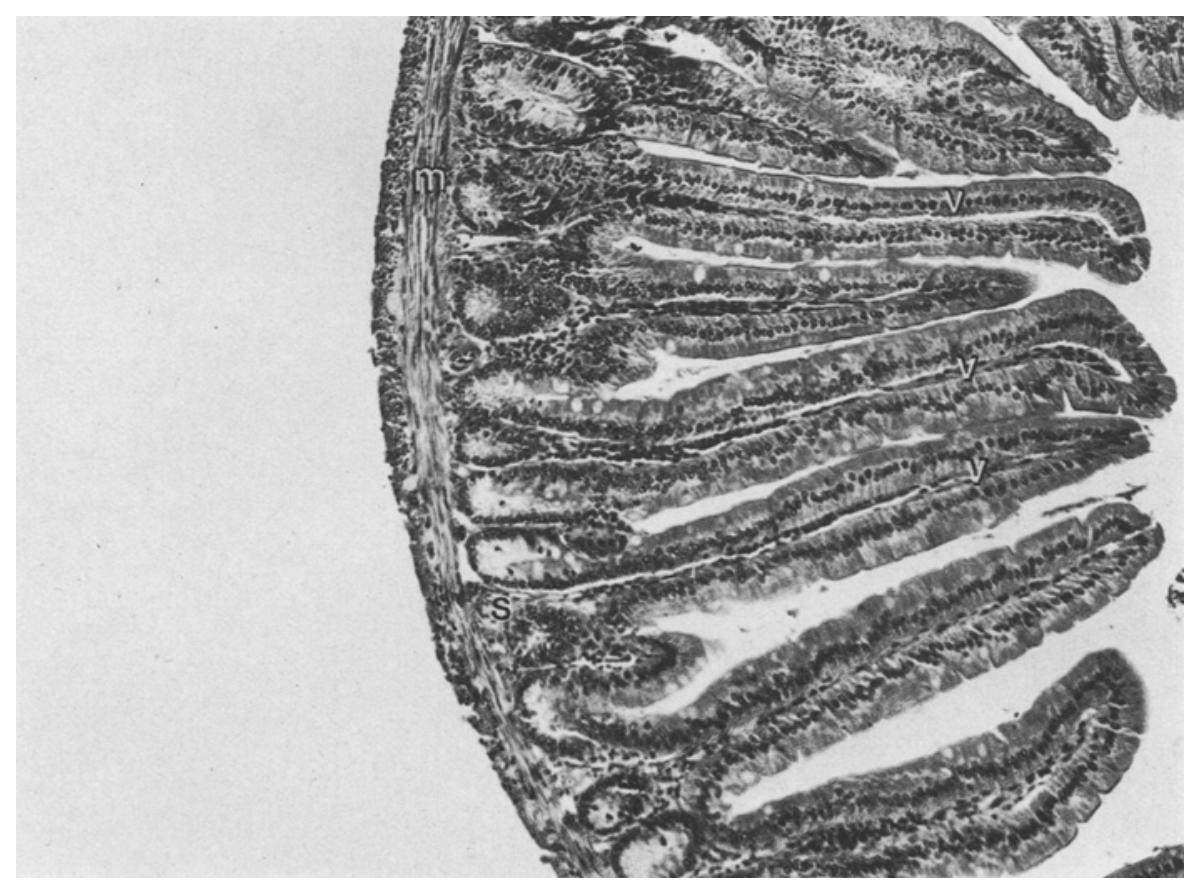

Fig. 1. Control intestine illustrating general gut morphology. Note muscularis (m), submucosa(s) and long, thin fingerlike villi (v). $100 \times$. Haematoxylin and eosin 


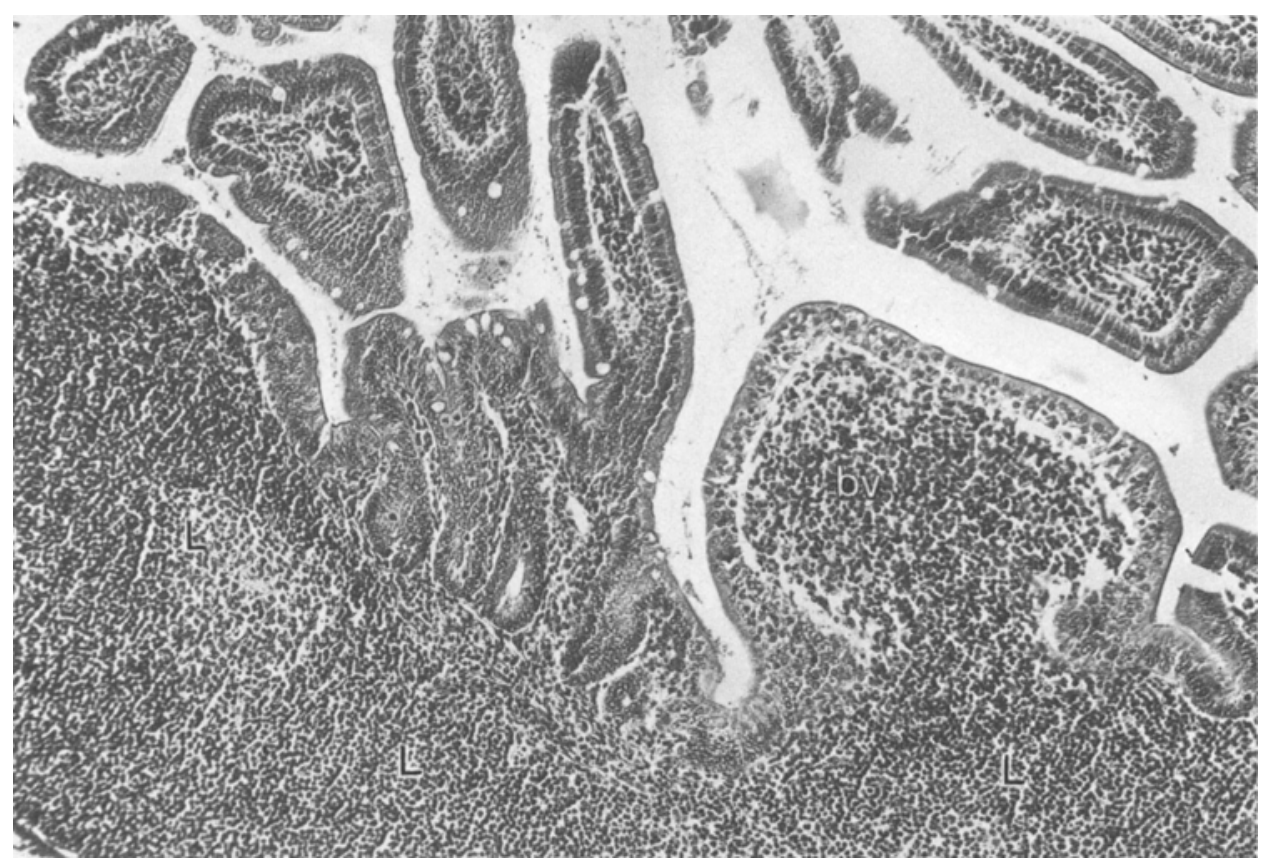

Fig. 2. Diabetic intestine illustrating a large lymphocyte aggregation ( $\mathrm{L}$ ) and blunted villus (bv). Note the thickening of mucosal and submucosal layers and the large area invaded by the lymphocytes. $100 \times$. Haematoxylin and eosin

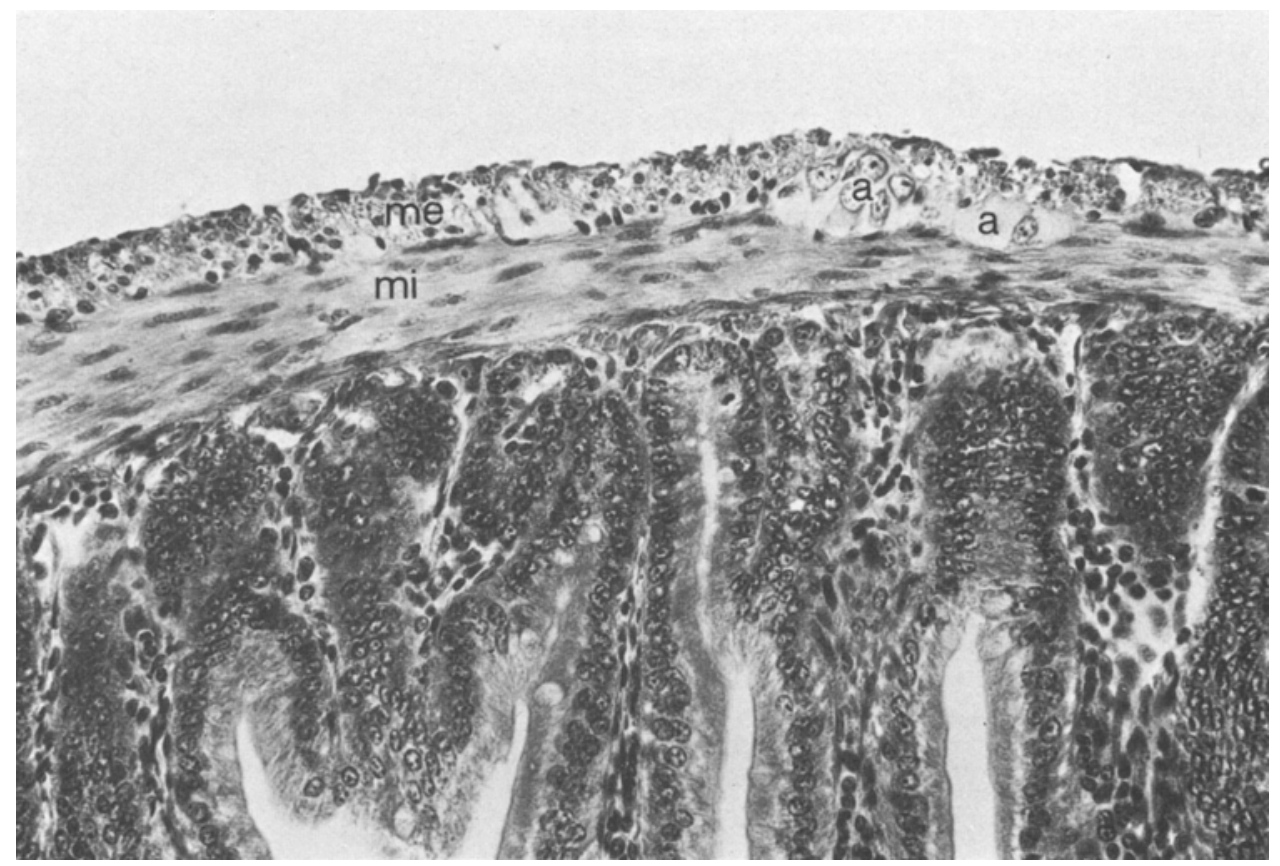

Fig. 3. Control intestine showing normal thickness of muscularis externa (me) and muscularis interna (mi) and rich supply of Auerbach's plexuses (a). $400 \times$. Haematoxylin and eosin 


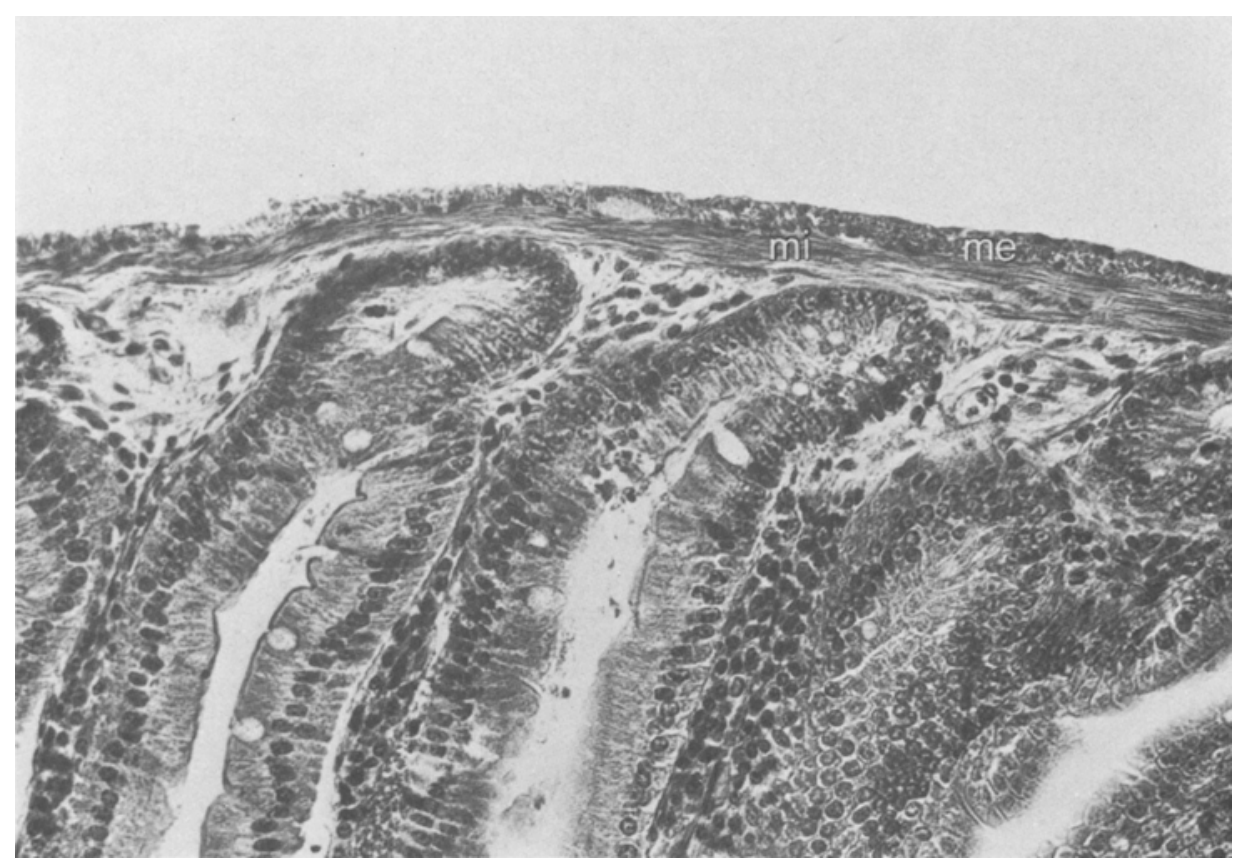

Fig. 4. Diabetic intestine showing reduction of muscularis externa (me) and muscularis interna (mi) and absence of Auerbach's plexuses. $400 \times$. Haematoxylin and eosin

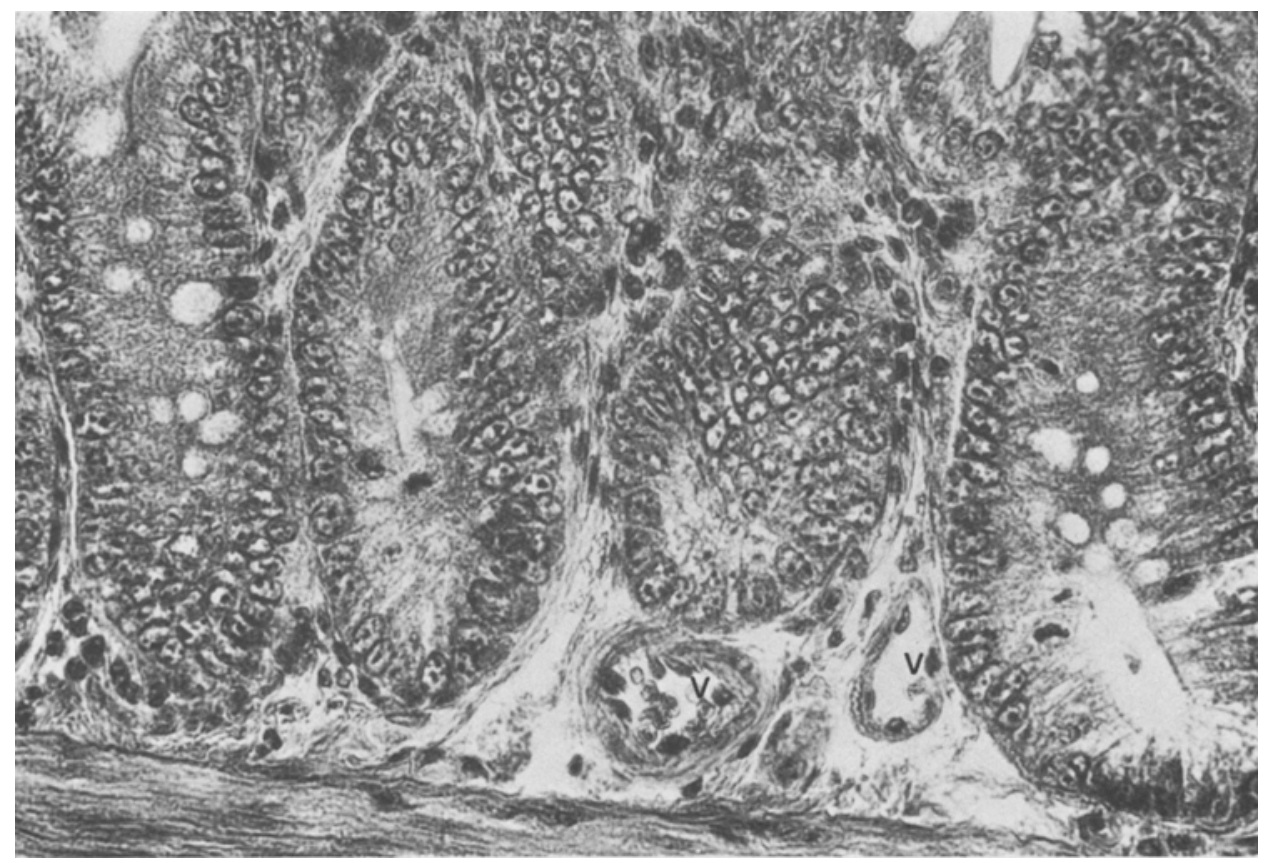

Fig. 5. Control intestine illustrating normal submucosal blood vessels (v). $400 \times$. Schorr's Trichrome 


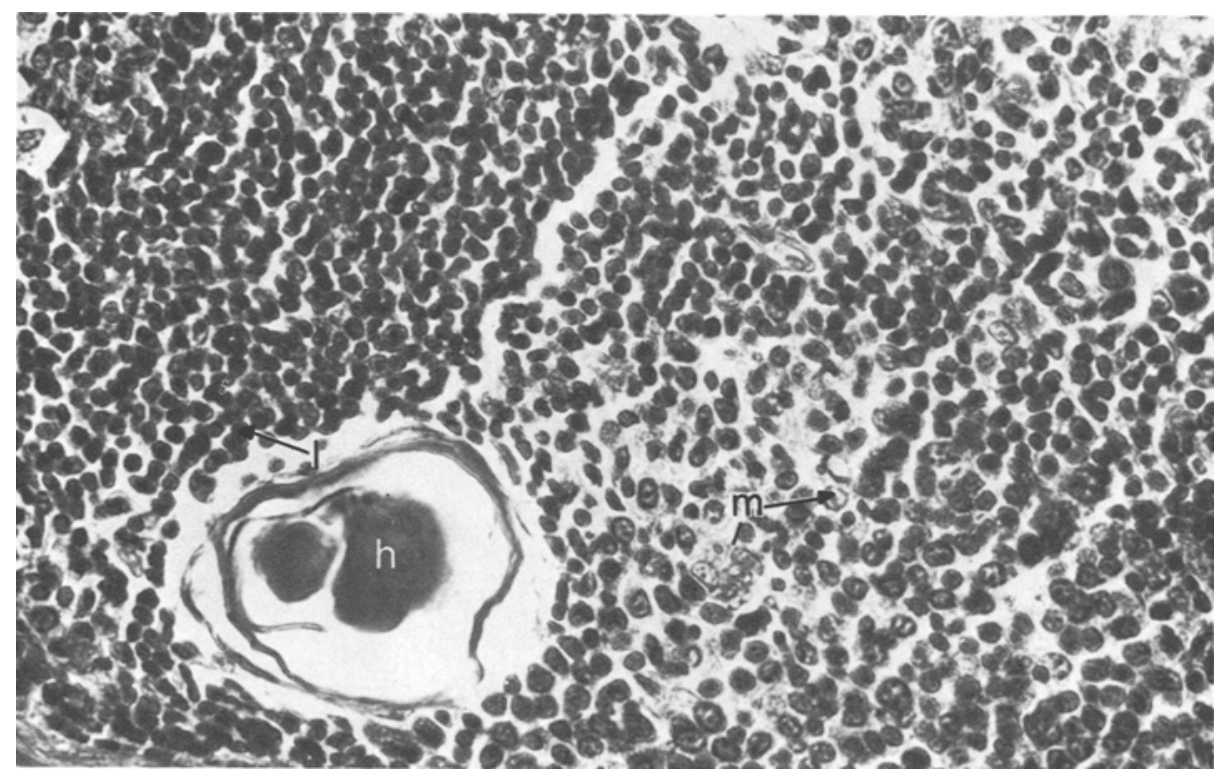

Fig. 6. Diabetic intestine illustrating a blood vascular lesion. Note hyaline deposit (h) in the lumen of the vessel, thickened and irregular vascular wall and numerous lymphocytes (1) and macrophages $(\mathrm{m})$ surrounding the vessel. $400 \times$. Schorr's Trichrome

Blood vessel dilation was restricted to the middle third of the diabetic intestines (Table 2). Although not shown, vasodilation was characterized by distension or enlargement of vessels. None of the other morphological changes, associated with blood vascular lesions described above, were observed in these dilated vessels.

Epithelial cell loss was prevalent in diabetic intestines compared with controls (Fig. 7), but was most pronounced in the middle third of the diabetic intestines (Table 2). This pathological condition can be described as excessive sloughing of cells from the villi (Fig. 8). It should be mentioned that the severity of this lesion was quite variable among the diabetics. For example, in some areas, only a few cells were lost from the tips of some of the villi whereas, in other regions, almost every villus was partially denuded of epithelium as shown in Fig. 8. This loss of epithelium resulted in deformation of the villi and exposed the tissue of the tunica propria to the luminal environment. Furthermore, massive amounts of debris, perhaps necrotic cells, were situated in the vicinity of the pathological villi. Examination of fresh intestinal tissues under the dissecting microscope also revealed epithelial cell pathology.

\section{Discussion}

An increase in the number of villi suggested that the diabetic intestine exhibited more luminal surface area than the controls. The length and width measurements of the villi also tended to support this hypothesis even though the differences were modest, but statistically significant. It should be pointed out that only normal appearing villi were measured. Thus, the differences may be considerably greater than suggested by the data. The changes observed in villi were consistent with the observation that increased intestinal mass may have enhanced fat transport in the diabetic Chinese hamster [6]. A more recent investigation reported that diabetic Chinese hamsters displayed an accelerated loss of carbohydrate calories through the urine and fecal material and a depressed absorption of dietary fat [7]. Data of the current study seemed to contradict those observations. However, epithelial loss from the villi might have attenuated the absorptive capacity of increased intestinal mass which could lead to reduced retention of ingested fat.

The increased intestinal mass and surface area may be related to the hyperphagia exhibited by the Chinese hamster. It is reasonable to assume that the previously reported hyperphagia [7] may promote some type of growth response in the gastrointestinal tract [20].

The enlarged cross-sectional area suggested distension of the diabetic gut. This may, in part, be due to accumulation of partially digested food. Similar distension has been reported for the human diabetic gastrointestinal tract $[16,21]$. It is possible that the enlarged cross sectional area may be related to hypertrophy, but this was not supported by a concomitant increase in muscle thickness.

Goblet cells functionally secrete mucus to protect 


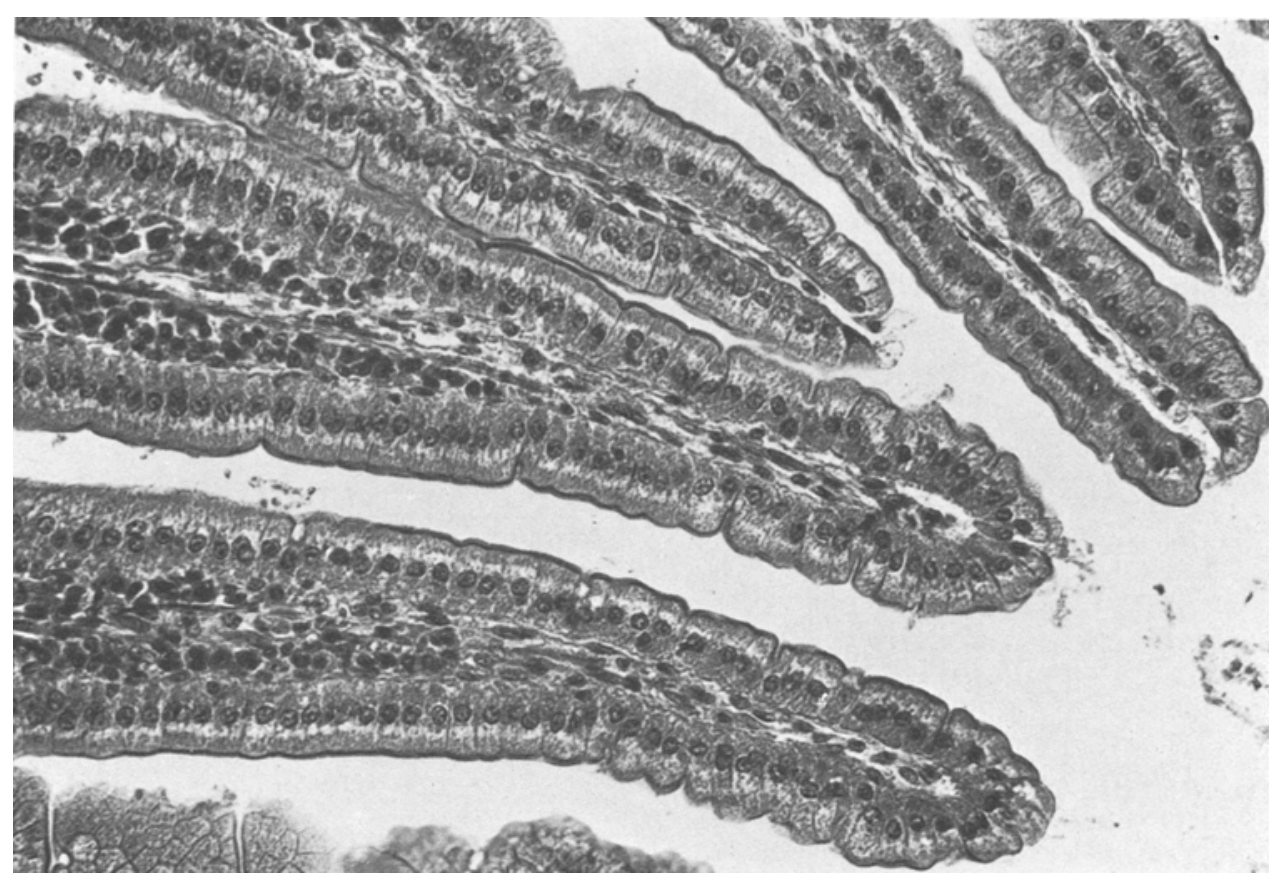

Fig. 7. Control intestine depicting normal villus structure. $400 \times$. Schorr's Trichrome

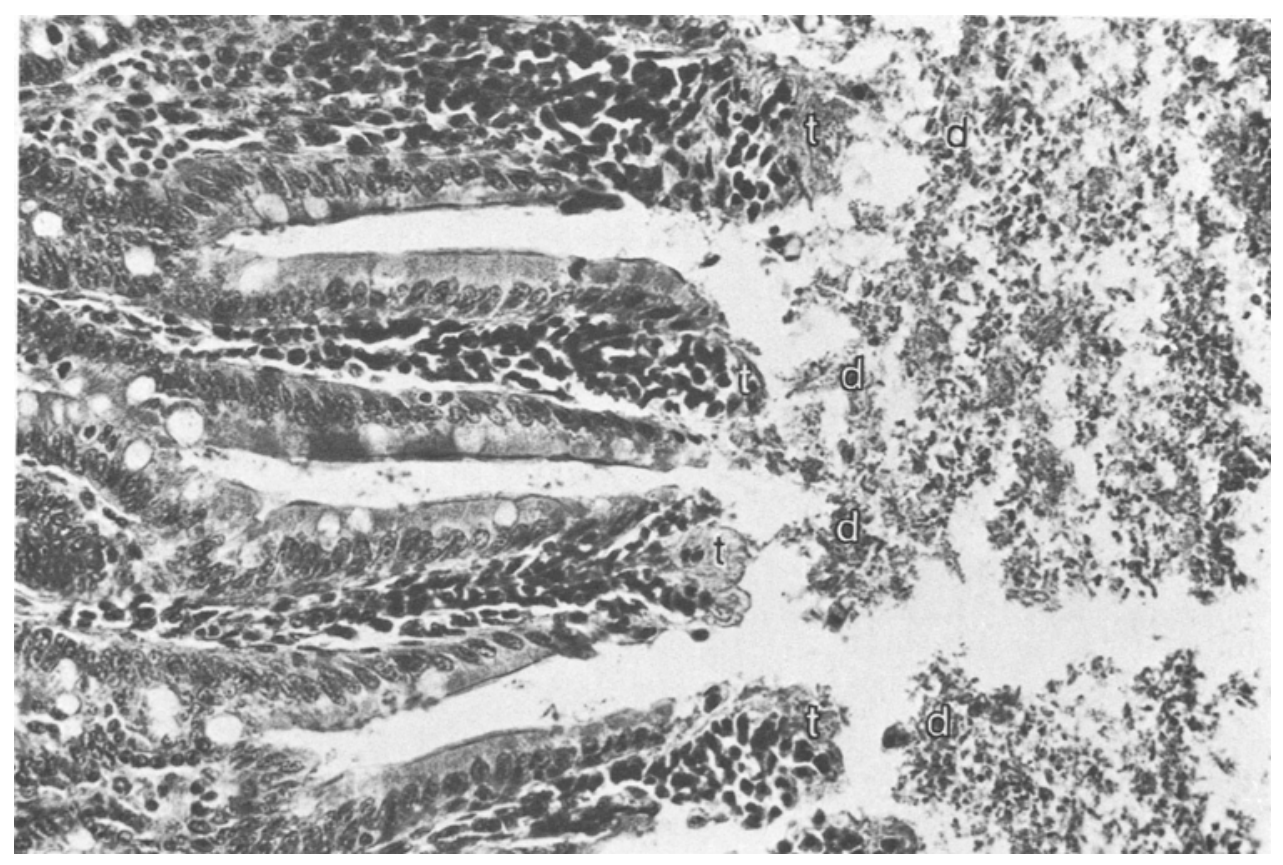

Fig. 8. Diabetic intestine depicting loss of epithelial cells from the villi. The villi (top of the figure and bottom of the figure) have lost almost half of their epithelial lining. Note the exposure of the tunica propria $(t)$ to the luminal environment and the cellular debris (d) near the villi. Note also the dense lymphocyte infiltration into the pathological villi. $400 \times$. Schorr's Trichrome 
the mucosal lining of the intestine from the abrasive character of food substances. Furthermore, it has been reported that increased quantity of food stimulates secretion of mucus [20]. Therefore, it was not surprising to find increased goblet cell numbers associated with hyperphagic Chinese hamsters.

The diminution of Auerbach's plexuses may be related to insufficient innervation of the muscularis layer. This could result in intestinal atony, thus explaining the friable intestine observed in the diabetic Chinese hamsters. Poor nervous supply of the muscularis might also lead to diminished segmentation, peristalsis and intestinal stasis, which could account for the accumulation of food observed in the diabetic intestines. Atony, distension, hypomotility and delayed emptying have all been seen in the gastrointestinal tract of human diabetics who displayed associated neuropathy $[16,17,21]$. It was also possible that inadequate innervation of the muscularis could have created a disuse atrophy thus leading to necrosis of the muscle fibers. Neuropathy has also been demonstrated in the distal peripheral nerves of the diabetic Chinese hamster [2]. In connection with the reduction of the muscularis was the presence of excessive connective tissue in the muscular coats of some of the diabetics. This observation might have intimated connective tissue replacement of pyknotic smooth muscle, but its occurrence was very infrequent. Connective tissue replacement of the outer longitudinal muscle layer has also been observed in diabetic man [22].

Shrinkage of cell bodies, pyknosis of nuclei and non-uniform staining of the neuroplasm have been reported in Auerbach's plexuses of human diabetics [22]. Similar changes have been observed in some diabetic Chinese hamsters.

The aetiology of the lymphocyte aggregations is unknown. It may be speculated that hyperphagia could irritate the small intestine, thereby inducing an inflammatory response. Bacterial overgrowth due to intestinal stasis could stimulate lymphocyte production. Elevated bacterial counts have been reported in the gastrointestinal tract of human diabetics [14]. It is of considerable interest to note that the blood vascular lesions were always associated with the lymphocyte aggregates. However, all lymphocyte aggregates did not have blood vascular lesions. It is therefore difficult to determine the cause and effect relationship, but it is possible that lymphocytes and macrophages may have phagocytized necrotic vascular tissues. Short, blunted villi were also seen in association with the lymphocyte aggregations. Similar abnormal villi and lymphocyte infiltration have been detected in human diabetics [16]. No hypotheses have been put forth concerning the pathological significance of blunted villi.

Blood vascular lesions were reported to contain hyaline material in the lumina of the vessels. This matter would presumably impede blood flow, thus creating a serious impediment to the process of intestinal assimilation and localized ischaemia. In addition to the hyaline deposit, deterioration of the vascular wall was observed which might result in plasma leakage. Plasma leakage might result in oedema and provoke lymphocyte infiltration. Notable thickening of the vascular wall could be indicative of a sclerotic condition, but sufficient data are not yet available to support this hypothesis.

Vasodilation was seen in areas of the intestine which were devoid of blood vascular lesions. This suggested that vasodilation was not related to the pathological vessels described above. The aetiology and ramifications of vasodilation are unclear.

The loss of epithelial cells might have altered the process of intestinal absorption. Without the presence of a brush border, active transport enzymes would be unavailable to aid absorption of nutrients. This loss of epithelium could possibly have manifested itself as a malabsorption syndrome similar to that shown in human diabetics [12]. The epithelial depletion and deformed villi might possibly suggest the presence of a sprue syndrome similar to that in human diabetics $[15$, 16]. The debris, near the denuded villi, was presumably of epithelial origin. It should be stated that epithelial cell loss from the tips of the villi is a normal phenomenon. Replacement of these lost cells is accomplished by mitosis and migration of cells from the crypts of Lieberkuhn [20]. It was plausible that the mitotic rate of the crypts was depressed in the intestines of some of the diabetics thus delaying epithelial cell replacement along the villi. It was also possible that the excessive loss of epithelial cells could have resulted from some type of basement membrane disorder.

The variability of some of the morphological changes reported may be due to several factors. Several highly inbred strains of Chinese hamsters were utilized in this study and it is possible that they have different genotypes which may mediate intestinal pathology [23]. Most hamsters used in the current investigation were diabetic for a short duration of time and, furthermore, were not severely diabetic. It has been shown that increased incidence of retinopathy and nephropathy were related to length and severity of diabetes in the Chinese hamster [1]. Variability of intestinal disorders have also been reported in human diabetics [12].

Acknowledgements. The authors wish to thank Dr. William Dulin for the professional advice and encouragement supplied during these investigations. Special 
thanks are also extended to Mr. Bill Burr and Mr. Ed Block of The Upjohn Company for photographic assistance.

\section{References}

1. Soret, M. G., Dulin, W. E., Mathews, J., Gerritsen, G. C.: Morphologic abnormalities observed in retina, pancreas, and kidney of diabetic Chinese hamsters. Diabetologia 10, 567-579 (1974)

2. Schlaepfer, W. W., Gerritsen, G. C., Dulin, W. E.: Segmental demyelination in the distal peripheral nerves of chronically diabetic Chinese hamsters. Diabetologia 10, 541-548 (1974)

3. Luse, S. A., Gerritsen, G. C., Dulin, W. E.: Cerebral abnormalities in diabetes mellitus: An ultrastructural study of the brain in early onset diabetes mellitus in the Chinese hamster. Diabetologia 6, 192-198 (1970)

4. Sirek, O. V., Sirek, A.: The colony of Chinese hamsters of the C. H. Best Institute: A review of experimental work. Diabetologia 3, 65-73 (1967)

5. McCombs, H. L., Gerritsen, G. C., Dulin, W. E., Chobanian, A. V.: Morphologic changes in the aorta of the diabetic Chinese hamster. Diabetologia 10, 601-606 (1974)

6. Parkinson, T. M.: Enhanced intestinal fat absorption in diabetic Chinese hamsters. Diabetologia 9, 505-509 (1973)

7. Gerritsen, G. C., Blanks, M.: Characterization of Chinese hamsters by metabolic balance, glucose tolerance and insulin secretion. Diabetologia 10, 493-499 (1974)

8. Frankel, B. J., Gerich, J., Hagura, R., Fanska, R., Gerritsen, G. C., Grodsky, G.: Abnormal secretion of insulin and glucagon by the in vitro perfused pancreas of the genetically diabetic Chinese hamster. J. clin. Invest. 53, 1637-1646 (1974)

9. Gerritsen, G. C., Dulin, W. E.: Characterization of diabetes in Chinese hamsters. Diabetologia 3, 74-78 (1967)

10. Bridwell, T., Whitehouse, F.: Peroral jejunal biopsy in patient with diabetic diarrhea: Case report. Diabetes 10, 58-59 (1961)

11. Drewes, V. M., Olson, S.: Histological changes in small bowel in diabetes mellitus: Study of peroral biopsy specimens. Acta path. microbiol. scand. 63, 478-480 (1965)
12. Ellenberg, M., Rifkin, H.: Diabetes mellitus: Theory and practice, p. 1031. New York: McGraw-Hill Book Company 1970

13. Finlay, J. M.: Small bowel complications of diabetes. Gastroenterology 48, 816 (1965)

14. Goldstein, F., Wirts, C., Kowlessar, O.: Diabetic diarrhea and steatorrhea, microbiologic and clinical observations. Ann. intern. Med. 72, 315-318 (1970)

15. Green, P. A., Wollaeger, E., Sprague, R., Brown, A.: Diabetes mellitus associated with nontropical sprue: Report of 4 cases. Diabetes 11, 388-392 (1970)

16. Katz, L. A., Spiro, H.: Gastrointestinal manifestations of diabetes. New Engl. J. Med. 275, 1350-1361 (1966)

17. McNally, E. F., Reinhard, A., Schwartz, P.: Small bowel motility in diabetics. Amer. J. dig. Dis. 14, 163-169 (1969)

18. Gerritsen, G. C., Dulin, W. E.: Interaction of genetics and environment on diabetes in the Chinese hamster as compared with human and other diabetic species. Acta diabet. lat. 9, 48-88 (1972)

19. Humason, G. L.: Animal tissue techniques, p. 468. San Francisco: W. H. Freeman and Company 1962

20. Guyton, A. C.: Basic human physiology: normal function and mechanisms of disease, p. 721. Philadelphia, London and Toronto: W. B. Saunders Company 1971

21. Rundles, R. W.: Diabetic neuropathy, general review with report of 125 cases. Medicine (Baltimore) 24, 111-160 (1945)

22. Berge, K. G., Sprague, R., Bennett, W.: The intestinal tract in diabetic diarrhea: A pathologic study. Diabetes 5, 289-294 (1956)

23. Gerritsen, G. C., Johnson, M. A., Soret, M. G., Schultz, J. R.: Epidemiology of Chinese hamsters and preliminary evidence for genetic heterogeneity of diabetes. Diabetologia 10, 581-588 (1974)

Received: September 9, 1975, and in revised form: December 2, 1975

A. R. Diani, Ph. D.

Dept. of Biology

Baylor Univ.

Waco, Texas 76703

USA 